\title{
Veinte frenéticos años de arqueología en la red y una pandemia para unirlos a todos
}

\author{
Jaime Almansa-Sánchez | Instituto de Ciencias del Patrimonio, Consejo Superior de Investigaciones Científicas (CSIC)
}

URL de la contribución <www.iaph.es/revistaph/index.php/revistaph/article/view/4792>

La web 1.0. fue un sistema unidireccional de información que permitió a finales del siglo XX crear una presencia digital con multitud de información estática que cualquiera podría consultar desde su casa con una conexión a internet. Algunas instituciones empezaron a sumarse, pero la infraestructura era aún costosa y el acceso limitado.

A principios del siglo XXI, la web 2.0 permitió un cambio de paradigma por el que el usuario podía interactuar con los contenidos y hacer realidad formas de diálogo y colaboración que hasta entonces se limitaban al correo electrónico y otros servicios de mensajería. Era el inicio de la red social y las conexiones cada vez eran más accesibles y rápidas. La adaptación llevó un tiempo, pero llegó también a las instituciones, menos amigas por lo general de esa bidireccionalidad.

La revolución llegó con el auge de las redes sociales y la web semántica, que algunos empezaron a llamar web 3.0, y nos abrió la puerta a un sinfín de posibilidades desde la palma de la mano y una accesibilidad casi total. Empezaba la dictadura del algoritmo y la democratización de Internet.

Para un arqueólogo, acostumbrado a manejar cronologías muy largas, ver que el gran paso de la web $1.0 \mathrm{a}$ la web semántica apenas llevó unos años (en España podemos ver el cambio abismal entre 2005 y 2010), es difícil no pensar en una verdadera revolución digital que abrió un mundo de posibilidades para la comunicación.

Mientras estaba en Londres (2007-2008), una compañera me pasó el enlace al programa de divulgación de The Prescott Street Dig (L-P Archaeology, 2008). Junto a actividades in situ, habían creado una web con comu- nicación en directo, el registro completo del yacimiento y miles de materiales educativos. Una quimera para mi experiencia anterior en España.

Pero el avance de la tecnología y la accesibilidad a los medios cambiaron de forma repentina el panorama. Ya no era necesario saber diseñar o programar, la plataforma venía dada y solo teníamos que subir los contenidos. El cambio con respecto a la comunicación en los medios tradicionales parecía evidente. La democratización del patrimonio arqueológico había llegado. Pero, incluso dejando de lado los aspectos más oscuros de la red (Morozov 2012), pronto se comenzaron a ver algunos detalles que podían poner en duda la verdadera efectividad del nuevo modelo y sus inconvenientes.

Aunque muchas de las redes sociales han servido más bien como medios de comunicación interna (entre profesionales), su potencial se ha visto pronto reflejado en el ámbito de la divulgación. Podemos ver ejemplos de acercamientos críticos a este potencial sobre Twitter (Laracuente 2012), Facebook (Rodríguez Temiño y González Acuña 2014) e incluso Trip Advisor (Corpas y Castillo 2019). Esta última red ha sido objeto de la última cuenta cómica que sigo en Instagram, compartiendo reseñas de una estrella sobre los grandes yacimientos arqueológicos del mundo y que ponen de manifiesto, no solo la necesidad de mediación, sino también lo cuestionable del modelo.

Durante más de diez años he trabajado con redes sociales, principalmente para proyectos y eventos arqueológicos. En un panorama que cambia en cuestión de meses, es difícil hacer un seguimiento pormenorizado de todo lo que sucede y, desde luego, generalizar es imposible. Hoy podemos encontrar principalmente cuatro perfiles 
en las redes sobre arqueología: 1) Divulgación (semi) profesional, con gran cantidad de seguidores y contenidos de cierta (a veces mucha) calidad; 2) Instituciones con presencia en las redes (unas veces amateur y otras profesional); 3) Profesionales con mayor o menor presencia online pero visibilidad limitada; y 4) Aficionados haciendo divulgación (por lo general de dudosa calidad).

Sin lugar a duda podemos hablar de una comunidad digital interesada en la arqueología. Dentro de ella, pequeñas comunidades de temas concretos, algunas casi exclusivamente profesionales, pero muchas de ellas abiertas a un amplio público. Ahora, ¿cuál es la diferencia con el mundo analógico? ¿Son realmente innovadoras?

Este año hice un pequeño sondeo para ver el impacto de la oferta digital durante el primer confinamiento de la covid-19 (Almansa-Sánchez 2020). Aún con sus limitaciones, algunas tendencias parecían claras:

> Lo digital no sustituye a lo presencial: aunque algunos perfiles, por lo general no tan entusiastas con la arqueología, se planteaban la capacidad de una buena reconstrucción virtual para reemplazar una visita en persona, la mayoría rechazaba este hecho.

> Apenas se amplía nuestra audiencia: seguimos dependiendo de redes preexistentes, forjadas presencialmente, que es difícil superar. Falta mucha investigación al respecto, pero no se ve un crecimiento fuera de los círculos ya cercanos.

> Sigue existiendo un público inaccesible: a pesar de la aparente permeabilidad y gran implantación de las redes sociales, aparte de las dificultades para ampliar audiencias, sigue habiendo grandes grupos que tampoco se harán eco del trabajo arqueológico en la red con las estrategias actuales.

Aunque hay también muchos aspectos positivos, y el potencial es evidente y está aún por explotar en gran medida, creo que básicamente seguimos reproduciendo los mismos esquemas de siempre, con poca innovación

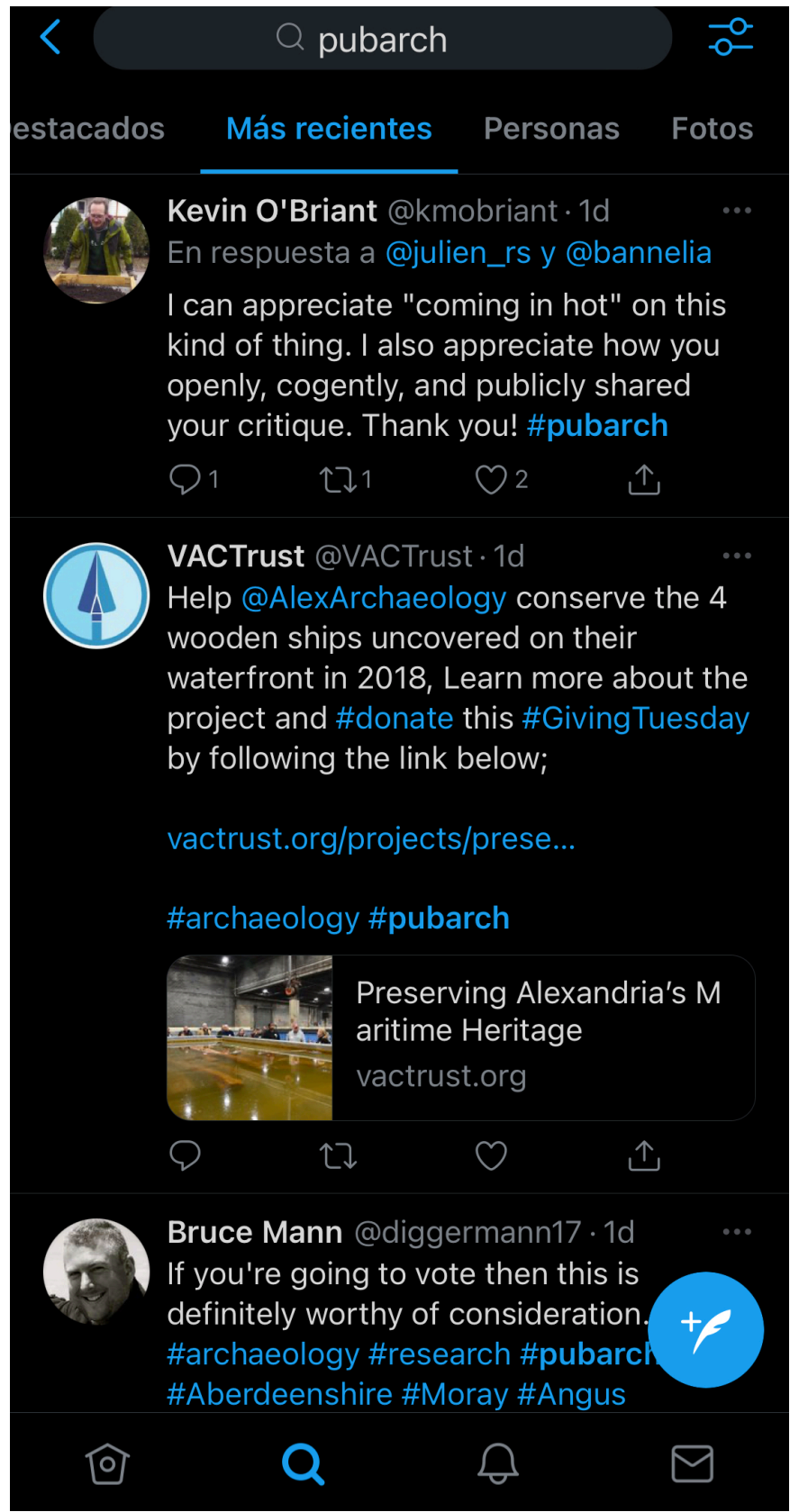

Pantallazo de las últimas discusiones en Twitter sobre arqueología pública

y un alcance poco mayor del que ya teníamos. Sigue siendo necesaria una mayor profesionalización de la comunicación (en general y virtual) y, sobre todo, innovación en formatos y contenidos. Ejemplos como el 
a debate Comunicación y redes sociales en instituciones culturales

| coordina Candela González Sánchez

fantástico Miguelón en Twitter de Antonio José Mencía para Atapuerca, o las puestas en escena queer de Puto Mikel en YouTube pueden ser reseñables. Pero, obviamente, la solución no es copiarlo, sino seguir innovando con todas las posibilidades que nos ofrece la tecnología, el indudable potencial del patrimonio y sin miedo a la supuesta banalización de la ciencia. Porque una buena divulgación no está reñida con lo performativo y tanto las nuevas tendencias comunicativas como los medios de difusión digitales hasta lo prefieren.

Ahora bien, en este tema, como en tantos otros, aún es necesario profundizar en la investigación desde una perspectiva multidisciplinar que nos permita conocer mejor las audiencias digitales y salvar los problemas que ya estamos identificando y que seguirán apareciendo.

\section{BIBLIOGRAFÍA}

- Almansa-Sánchez, J. (2020) Visitas virtuales durante el confinamiento de la covid-19. Digital CSIC. Disponible en línea: http://dx.doi.org/10.20350/digitalCSIC/12684 [Consulta: 04/12/2020]

- Corpas, N. y Castillo, A. (2019) Tourism 3.0 and archaeology: approaching tourists' generated-content of World Heritage sites. Pasos. Revista de Turismo y Patrimonio Cultural, vol. 17, n. ${ }^{\circ}$ 1, pp. 39-52. Disponible en: https://doi.org/10.25145/j. pasos.2019.17.003 [Consulta: 04/12/2020]

- L-PArchaeology (2008) Precott Street. Disponible en: https:// www.Iparchaeology.com/prescot/ [Consulta: 01/12/2020]

- Laracuente, N. (2012) Public Archaeology 2.0. Facilitating engagement with Twitter. AP: Online Journal in Public Archaeology, n. ${ }^{\circ}$ 2, pp. 81-99. Disponible en: http://dx.doi. org/10.23914/ap.v2i0.15 [Consulta: 04/12/2020]

- Morozov, E. (2012) The net delusion. The dark side of internet freedom. New York: Public Affairs

- Rodríguez Temiño, I. y González Acuña, D. (2014) Using Facebook to build a community in the Conjunto Arqueológico de Carmona (Seville, Spain). AP: Online Journal in Public Archaeology, n. ${ }^{\circ}$ 4, pp. 61-94. Disponible en: http://revistas. jasarqueologia.es/index.php/APJournal/article/view/44 [Consulta: 04/12/2020] 